\title{
El análisis crítico de la información publicada en la literatura médica
}

\author{
Tomás Pantoja C $\mathbf{1}^{\mathbf{1}}$, Luz María Letelier $\mathbf{S}^{\mathbf{2}, 4}$, \\ Ignacio N eumann $B^{3,4}$
}

\section{A critical appraisal of clinical reports in medical journals}

E nfrentado a la inmensa cantidad de información publicada en la literatura médica respecto a preguntas clínicas específicas, con resultados muchas veces contradictorios, es necesario disponer de herramientas que permitan analizar en forma crítica esa información. Dichas herramientas habitualmente consisten en una serie de criterios que intentan responder preguntas específicas de acuerdo al tipo de estudio que estamos analizando ${ }^{1-3}$. A su vez, el tipo de estudio está relacionado con la pregunta clínica que generó el inicio del proceso de búsqueda de información ${ }^{4}$. Sin embargo, independiente del tipo de estudio, este sanálisis crítico» está estructurado habitualmente en tres secciones que analizan diferentes aspectos del estudio:

1. La validez interna del estudio: sección que analiza si el diseño es apropiado para responder la pregunta clínica y si los métodos utilizados evitaron o disminuyeron al máximo la posibilidad de obtener un resultado «sesgado».

2. Los resultados del estudio: sección que analiza los resultados propiamente tales, la forma de expresarlos de acuerdo con el diseño y la pregunta escogida, y la precisión de ellos.
3. La aplicabilidad o validez externa del estudio: sección que analiza si los resultados son aplicables a la situación clínica particular que generó inicialmente la pregunta.

Es decir, cada sección está enfocada en un aspecto particular del estudio y su conexión con la pregunta inicial. A continuación describimos en mayor detalle dichos aspectos.

\section{LA VALIDEZ INTERNA: ¿ES VÁLIDO EL ESTUDIO?}

Un primer aspecto general es lo apropiado del diseño elegido para responder la pregunta planteada. No todas las preguntas clínicas son respondidas adecuadamente por ensayos clínicos randomizados (ECR) ni por revisiones sistemáticas. Si bien dichos diseños tienen un rol claramente establecido en el ámbito de la terapia, son otros los diseños utilizados para aproximarse a las preguntas de pronóstico o para evaluar la precisión de tests diagnósticos ${ }^{4}$.

Un aspecto central de la validez interna de los estudios es la medida en que los autores fueron

\footnotetext{
${ }^{1}$ Departamento de Medicina Familiar, Pontificia Universidad Católica de Chile.

${ }^{2}$ Departamento de Medicina Interna, Pontificia Universidad Católica de Chile. Servicio de Medicina, Hospital Sótero del Río.

${ }^{3}$ Residente de Medicina Interna, Departamento de Medicina Interna, Pontificia Universidad Católica de Chile.

4Unidad de Medicina Basada en Evidencia, Pontificia Universidad Católica de Chile.
}

Correspondencia a: Dr. Tomas Pantoja C. Unidad de Medicina Basada en Evidencia, Universidad Católica de Chile. E mail: umbeuc@med.puc.cl 
capaces de evitar el «sesgo». Este concepto está fuertemente relacionado a lo que conocemos como error, considerando la diferencia existente entre sesgo y error aleatorio:

- Error aleatorio o por azar: es aquel inherente a la naturaleza de la observación y difícilmente puede ser evitado. Dado que se presenta por azar, es esperable que se distribuya en forma balanceada entre diferentes grupos, por lo que generalmente no influye en los resultados de manera importante. Una manera de minimizarlo es aumentando el tamaño muestral (número de pacientes incluidos en el estudio).

- Error sistemático o sesgo: es aquél que se produce sistemáticamente en la misma dirección y dado que no se presenta por azar, es esperable que se distribuya asimétricamente en los diferentes grupos, y afecte los resultados. Existen diferentes tipos de sesgo en los diferentes tipos de estudio, de acuerdo al origen del «desbalance» entre los grupos.

Ambos tipos de error se representan gráficamente en la Figura 1.

De esta manera, un estudio será válido en la medida que el diseño elegido sea apropiado para responder el tipo de pregunta planteada y los métodos del estudio logren minimizar la posibilidad de sesgo en los resultados.

\section{LOS RESULTADOS DEL ESTUDIO: ¿CUÁLES SON LOS RESULTADOS?}

Los resultados del estudio pueden ser presentados de diversas maneras por los autores, dependiendo del tipo de pregunta y tipo de estudio. Es así como, en el caso de los ECR, los resultados de la comparación entre dos grupos respecto a un resultado medido dicotómicamente pueden ser expresados en términos absolutos, a través de la reducción del riesgo absoluto (RRA) o el número necesario para tratar (NNT), o en términos relativos a través de la reducción de riesgo relativo (RRR), el riesgo relativo (RR) 0 el odds ratio (OR). Los lectores debemos ser capaces de conocer las diferentes maneras de presentación de los resultados y de interpretarlos más allá de la mera significación estadística, considerando siempre la situación que originalmente planteó la pregunta. De esta manera, no sólo necesitamos saber los resultados en términos cualitativos (ctiene algún efecto la droga $\mathrm{X}$ comparada con placebo o con la droga $Y$ ?, isirve el test $\mathrm{X}$ para el diagnóstico de la condición $Y$ ?), sino también una estimación cuantitativa de los resultados y su grado de variabilidad o dispersión (¿cuál es la reducción de riesgo absoluto para un resultado $\mathrm{Z}$ de la droga $\mathrm{X}$ comparada con la droga Y? ¿cuáles son los intervalos de confianza de dicha reducción de riesgo?).

Así, debemos ser capaces de reconocer la forma cómo los resultados fueron presentados, e interpretarlos en su magnitud y variabilidad de acuerdo a la situación clínica.

\section{LA VALDEZ EXTERNA:}

¿CUÁN APLICABLE SON ESTOS RESULTADOS A LA SITUACIÓN CLÍNICA QUE ORIGINÓ LA PREGUNTA?

Finalmente, luego del análisis de la validez interna y la interpretación de los resultados, está el tema de cuán aplicables son los resultados a mi paciente en particular. Dentro de los aspectos a considerar en esta sección deben estar:

- ¿Cuán diferente es mi paciente de aquellos incluidos en el estudio? En casi cualquier

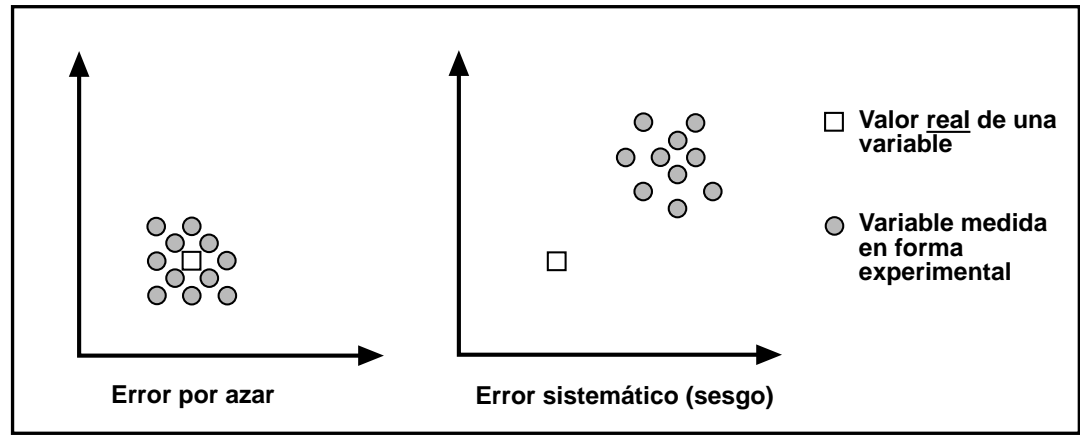

FiguRA 1. Representación gráfica de los diferentes tipos de emor en la investigación clínica. 
situación mi paciente no será totalmente similar a los del estudio. Sin embargo, el análisis debiera ser realizado desde la perspectiva de si mi paciente es tan diferente como para que los resultados no sean aplicables. De la misma manera, deben ser considerados aquellos aspectos del sistema de salud que puedan ser tan diferentes que impidan la implementación de la intervención o que los costos sean prohibitivos para nuestro sistema sanitario. Es decir, deben considerarse todos aquellos aspectos del contexto que puedan influir en la decisión final.

- Un segundo aspecto es aquel relacionado con el hecho de si se consideraron todos los resultados relevantes para la toma de decisiones. Para ello debe volverse atrás a la pregunta y pensar en cuáles eran los resultados de interés en la situación clínica inicial. Muchas veces los autores incluyen resultados que, pudiendo ser relevantes desde la perspectiva del investigador (variables fisiológicas, hemodinámicas, etc.), no lo son desde el punto de vista del médico clínico y su paciente (alivio del dolor, disminución de la mortalidad, etc.).

\section{REFERENCIAS}

1. Oxman AD, Sackett DL, Guyatt GH, for the Evidence-Based Medicine Working Group. Users' Guide to the Medical Literature I. How to get started? JAMA 1993; 270: 2093-5.

2. Sackett DL, Straus SE, Richardson WS, Rosenberg WMC, HAYNES RB. Evidence-Based Medicine. How
- Finalmente, debe realizarse un balance entre los efectos «positivos»de determinada intervención y los efectos negativos» (costo, efectos adversos, etc.). Aquí la pregunta es si los beneficios obtenidos por la intervención son suficientes para justificar los costos y efectos adversos. Esto representa una apreciación subjetiva, tanto del médico como de su paciente, $\mathrm{y}$ es aquí donde debieran considerarse los valores del paciente, su familia y el médico en el proceso de toma de decisiones.

\section{CONCLUSIÓN}

Lo expuesto representa una aproximación genérica al análisis crítico de la información publicada en la literatura médica. De acuerdo al tipo de pregunta y estudio deberán analizarse aspectos específicos de los métodos o la presentación e interpretación de los resultados, los que serán detallados en futuros artículos de esta Sección.

to Practice and Teach EBM? 2nd ed. Edinburgh: Churchill Livingstone, 2000.

3. Guyatt GH, RenNIE D. Users' Guides to the Medical Literature. A Manual for Evidence Based Clinical Practice. Chicago: AMA Press, 2002.

4. Sото M, Rada G. Medicina Basada en Evidencia: Formulación de preguntas. Rev Méd Chile 2003; 131: 1202-3. 